\title{
E-SECO ProVersion: Manutenção e Evolução de Experimentos Científicos
}

\author{
Tassio F. M. Sirqueira', ${ }^{1,2}$ Humberto L. O. Dalpra', Regina Braga ${ }^{1}$, \\ Marco A. P. Araújo ${ }^{1,2}$, José Maria N. David ${ }^{1}$, Fernanda Campos ${ }^{1}$ \\ ${ }^{1}$ Programa de Pós-graduação em Ciência da Computação - \\ Departamento de Ciência da Computação - Universidade Federal de Juiz de Fora \\ (UFJF) - Juiz de Fora - MG - Brasil \\ ${ }^{2}$ Instituto Federal de Educação, Ciência e Tecnologia do Sudeste de Minas Gerais \\ (IF Sudeste MG) - Campus Juiz de Fora - Juiz de Fora - MG - Brasil \\ \{tassio.sirqueira, marco.araujo\}@ice.ufjf.br, humbertodalpra@gmail.com \\ \{regina.braga, jose.david, fernanda.campos\}@ufjf.edu.br
}

\begin{abstract}
This paper discusses some characteristics of a scientific software ecosystem and the life cycle of an experiment, with emphasis on maintenance and evolution aspects. The E-SECO ProVersion is presented, that aim to support maintenance and evolution of experiments using data provenance. An extension of PROV model is presented together with an ontology, named PROV-OEXT. The article also presents existing workflows repositories and discuss the support that they provide to evolution and maintenance.
\end{abstract}

Resumo. Este trabalho discute algumas características de um ecossistema de software científico e do ciclo de vida de um experimento, com ênfase em manutenção e evolução dos experimentos, usando dados de proveniência. Uma extensão do modelo PROV é apresentada conjuntamente com uma ontologia, PROV-OEXT. O artigo também apresenta repositórios de workflows existentes e discute o suporte provido por esses no que tange a manutenção e evolução.

\section{Introdução}

Um experimento científico pode ser definido como um conjunto de atividades (análises) interligadas entre si [GOBLE et al., 2010]. O ciclo de vida de um experimento é composto por diversas etapas, desde a concepção do problema até a obtenção dos resultados conforme proposto por Belloum et al. (2011). Todas essas etapas do experimento devem ser registradas de forma a manter o registro da modelagem $\mathrm{e}$ execução do mesmo.

Um workflow científico é um modelo ou template que representa a sequência de atividades implementadas por ferramentas, programas ou serviços [DEELMAN et al., 2009]. Um ou mais workflows científicos podem ser utilizados para a execução de um experimento. Workflows científicos são interpretados e executados por Sistemas Gerenciadores de Workflows Científicos (SGWfC). No geral, os SGWfC limitam-se a gerenciar a execução de workflows científicos de forma isolada ao experimento do qual fazem parte. Assim, de acordo com Hasan et al. (2007), faz-se necessário o uso de ferramentas independentes do SGWfC para apoiar o desenvolvimento do experimento 
científico, sendo necessário o registro das variações dos workflows associados ao experimento, devido as modificações no decorrer da pesquisa [MATTOSO et al., 2009].

Neste contexto, podemos considerar que todas as informações a respeito do experimento científico fazem parte de sua gerência de configuração [MATTOSO et al., 2009]. Assim, avanços e mudanças no experimento devem ser registrados, criando uma base de conhecimento sobre a pesquisa. O registro dessas informações pode se tornar ainda mais útil se aplicado ao conceito de laboratórios colaborativos [VAZ et al., 2012], onde pesquisadores, geograficamente dispersos, estão trabalhando em um mesmo experimento e as atividades e dados de análises devem ser registrados e compartilhados com os demais pesquisadores do grupo, evitando a perda de conhecimento e do controle sobre os dados do experimento. Para isso, é necessário o armazenamento tanto dos dados quanto dos processos que os geraram. Uma das abordagens para se realizar esse registro é o uso de modelos de proveniência [MATTOSO et al., 2009].

Alguns SGWfC como o Taverna [OINN et al., 2007], Kepler [ALTINTAS et al., 2004] e Pegasus [GIL et al., 2007], permitem capturar os passos do workflow (processo e dados) durante sua execução. Esses sistemas, em geral, adotam modelos proprietários para capturar os traços de proveniência gerados nas execuções. Com a ausência da padronização entre os SGWfC é difícil a interoperabilidade dos dados, bem como a consulta e a análise pelo pesquisador. Faz-se necessário um modelo de proveniência de dados que permita a captura de proveniência retrospectiva e prospectiva, mantendo o padrão para qualquer SGWfC. Proveniência de dados é o registro da história da derivação dos dados, que possibilita a reprodutibilidade, interpretação dos resultados e diagnóstico de problemas [LIM et al., 2010]. Estas informações podem ser usadas para identificar métodos, regras, auxiliar os usuários na criação de workflows semelhantes, na compreensão de correlações de dados e na experiência para futuros experimentos [MOREAU et al., 2011]. Neste trabalho é utilizado o modelo de proveniência de dados PROV [MOREAU e MISSIER, 2013], padronizado pela W3C.

Todos os dados referentes ao experimento fazem parte de sua gerência de configuração, a qual deve acompanhar todos os passos da pesquisa, utilizando-se da proveniência de dados. Além desta, informações como derivações, manutenções e evolução do experimento e workflow vinculados, contribuem para a compreensão do mesmo. Para apoio ao respectivo controle, pode-se utilizar, como arcabouço, as técnicas de manutenção e evolução de software.

Assim, propõe-se o desenvolvimento de uma arquitetura para suporte a manutenção e evolução de experimentos científicos, fornecendo, de maneira automatizada, informações estratégicas relacionadas a evolução e manutenção do experimento, de forma que os cientistas possam tomar decisões ou obter maior conhecimento em relação ao mesmo. Esta arquitetura denomina-se E-SECO ProVersion, e é parte da abordagem E-SECO [FREITAS et al., 2015]. O E-SECO ProVersion busca adicionar funcionalidades específicas para a gerência de configuração de experimentos científicos no contexto de ecossistemas de software científicos e de laboratórios colaborativos

O artigo é composto por 3 seções, além da introdução. A seção 2 apresenta os trabalhos relacionados. Na seção 3 é apresentada a proposta do E-SECO ProVersion, 
sua arquitetura, seu desenvolvimento e uma breve análise junto a base do myExperiment. Por fim, a seção 4 apresenta as conclusões e os trabalhos futuros.

\section{Trabalhos Relacionados}

O SimiFlow é uma arquitetura para comparação e agrupamento de workflows préexistentes, por similaridade, visando a construção de vários experimentos por meio de abordagem ascendente [SILVA et al., 2010]. O CollabCumulus é um portal com conteúdo de repositórios de proveniência que realiza a análise dos dados gerados ou consumidos [MIRANDA et al., 2014]. O PBASE [CUEVAS-VICENTTÍN et al., 2014] é uma extensão do modelo de proveniência PROV destinada a workflows científicos, permitindo análise e replicação de experimentos.

O myExperiment é um ambiente colaborativo de compartilhamento e publicação de workflows [GOBLE et al., 2010]. O CrowDLabs é um repositório similar que foi desenvolvido pelo grupo do SGWfC VisTrails [CALLAHAN et al., 2006]. Permite a execução de workflows, importação de dados e reutilização por terceiros, porém não permite a análise detalhada de seus dados.

O diferencial da proposta do E-SECO ProVersion em relação a estes trabalhos é o suporte explícito a manutenção e evolução de experimentos científicos. Para isso utiliza uma base de dados de proveniência, modelada segundo o padrão PROV, que garante a interoperabilidade dos dados entre experimentos. Por meio de uma ontologia, apoio de regras de inferência para a descoberta de informações implícitas, e o acesso à repositórios de workflows, objetiva-se a formação de uma base histórica de dados de experimentos, com vistas a um melhor suporte a manutenção e evolução dos mesmos.

\section{E-SECO ProVersion}

O uso de workflows científicos é uma abordagem bastante utilizada no contexto de eScience e existem muitas pesquisas voltadas para o gerenciamento e execução de experimentos baseados em workflows. No entanto, experimentos complexos envolvem interações entre pesquisadores geograficamente distribuídos, podendo caracterizar-se como laboratórios colaborativos, e que demandam a utilização de grandes volumes de dados, serviços e recursos computacionais distribuídos. Este cenário categoriza um ecossistema de experimentação científica [FREITAS et al., 2015].

Neste contexto, foi proposta uma abordagem baseada em ecossistemas de software [BOSCH, 2009], denominada E-SECO (E-Science Software ECOsystem) [FREITAS et al., 2015]. O E-SECO possui um ciclo de vida baseado na proposta de Belloum et al. (2011). Este ciclo de experimentação foi expandido para englobar a abordagem E-SECO ProVersion, a fim de viabilizar o suporte a gerência de configuração de experimentos, conforme Figura 1.

A gerência de configuração deve ser entendida como uma etapa de suma importância para o ciclo de vida de um experimento e dos workflows vinculados. Através desta pode-se determinar o estado do experimento em um determinado momento, o que, considerando o contexto de um laboratório colaborativo, pode derivar informações importantes para acertos futuros, tais como resultados parciais, erros encontrados ou gerados, entre outras informações, durante a execução do experimento. 
Além disso, permite-se prever o comportamento do experimento no futuro e, no caso dos workflows, como os mesmos devem ser mantidos e evoluídos.

Conforme detalhado por Mattoso et al. (2009), o apoio a reutilização e a gerência de configuração deve ser tratado com um item importante a ser explorado, de forma a diminuir o retrabalho e apoiar o aumento de produtividade e de qualidade dos experimentos dos pesquisadores. São necessárias soluções mais abrangentes, que permitam tratar as manutenções e evoluções dos workflows ao longo do seu ciclo de experimentação e permitir a reutilização do próprio experimento.

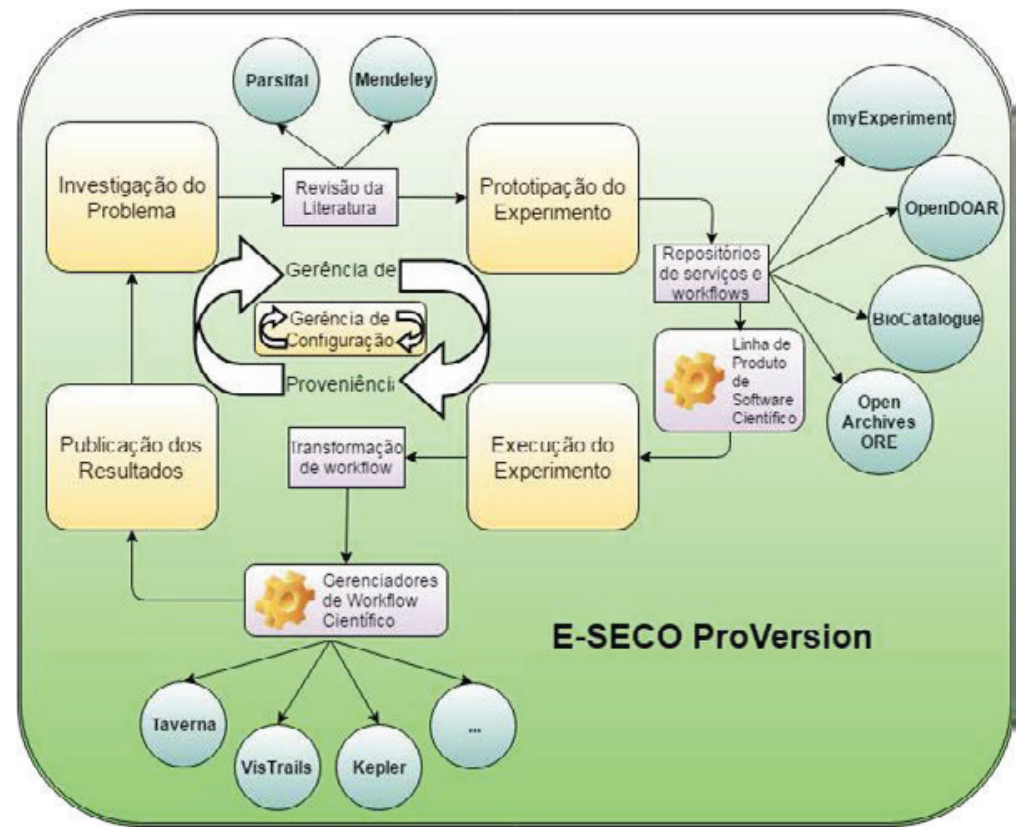

Figura 1. Ciclo de vida de um experimento científico no E-SECO ProVersion.

$\mathrm{Na}$ proposta E-SECO ProVersion, a gerência de configuração de um experimento está dividida em duas etapas, Gerência de Proveniência e Gerência de Manutenção e Evolução. A Figura 2 apresenta a arquitetura do E-SECO ProVersion, integrando os módulos relacionados à gerência de configuração. $O$ gerente de manutenção e evolução é responsável pelo controle de dados de proveniência prospectiva e retrospectiva de um experimento, com o objetivo de controlar a evolução e as manutenções existentes no experimento e workflows a ele vinculado, além disso, considera que o experimento pode ser composto por múltiplos workflows, e para cada um são geradas diversas versões que devem ser controladas. Além das informações do experimento a qual faz parte, informações acerca do comportamento do workflow, resultados de execução e controle dos dados consumidos e gerados no experimento também são controlados pelo módulo. O módulo de proveniência, também pertencente ao gerente de configuração, é responsável pela coleta e armazenamento dos dados capturados durante a execução dos workflows, que são utilizados pelo módulo de manutenção e evolução. Como o E-SECO ProVersion está inserido no contexto de um ecossistema e a interoperabilidade dos dados é um fator importante, o uso de um modelo de proveniência padrão, amplamente aceito pela comunidade, é importante. Neste sentido, o modelo PROV (MOREAU \& MISSIER, 2013) foi o escolhido para ser utilizado na proposta deste trabalho, compondo a gerência de proveniência. A modelagem do banco, a qual segue as regras do modelo PROV, pode ser acessada no 
seguinte endereço http://goo.gl/LvP8Aq. Este modelo de proveniência permite acompanhar as etapas do experimento, segundo o modelo de ciclo de vida definido, criando uma base de conhecimento sobre a pesquisa. Além da coleta e o armazenamento em uma base de dados, uma ontologia integrada ao módulo é utilizada, juntamente com os dados do experimento, para auxiliar na extração do conhecimento por meio de inferências, possibilitando a identificação de workflows com tarefas similares, fluxos de trabalho próximos e características de manutenção e evolução.

O modelo PROV já conta com o suporte de uma ontologia denominada PROVO [LEBO et al., 2013], que é pública e disponibilizada pela W3C. No entanto, a PROVO não expressa todo o conhecimento necessário para o suporte a evolução e manutenção de workflows e experimentos. O trabalho de expansão da ontologia PROV-O, através da criação da ontologia PROV-OEXT, permite a descoberta de novas informações, tanto para a captura da proveniência prospectiva quanto retrospectiva. Algumas das relações causais da ontologia PROV-OEXT são exibidas na Figura 3. Com essas informações, o E-SECO ProVersion pode sugerir mudanças estratégicas tanto na modelagem quanto na execução do experimento ou workflows associados.

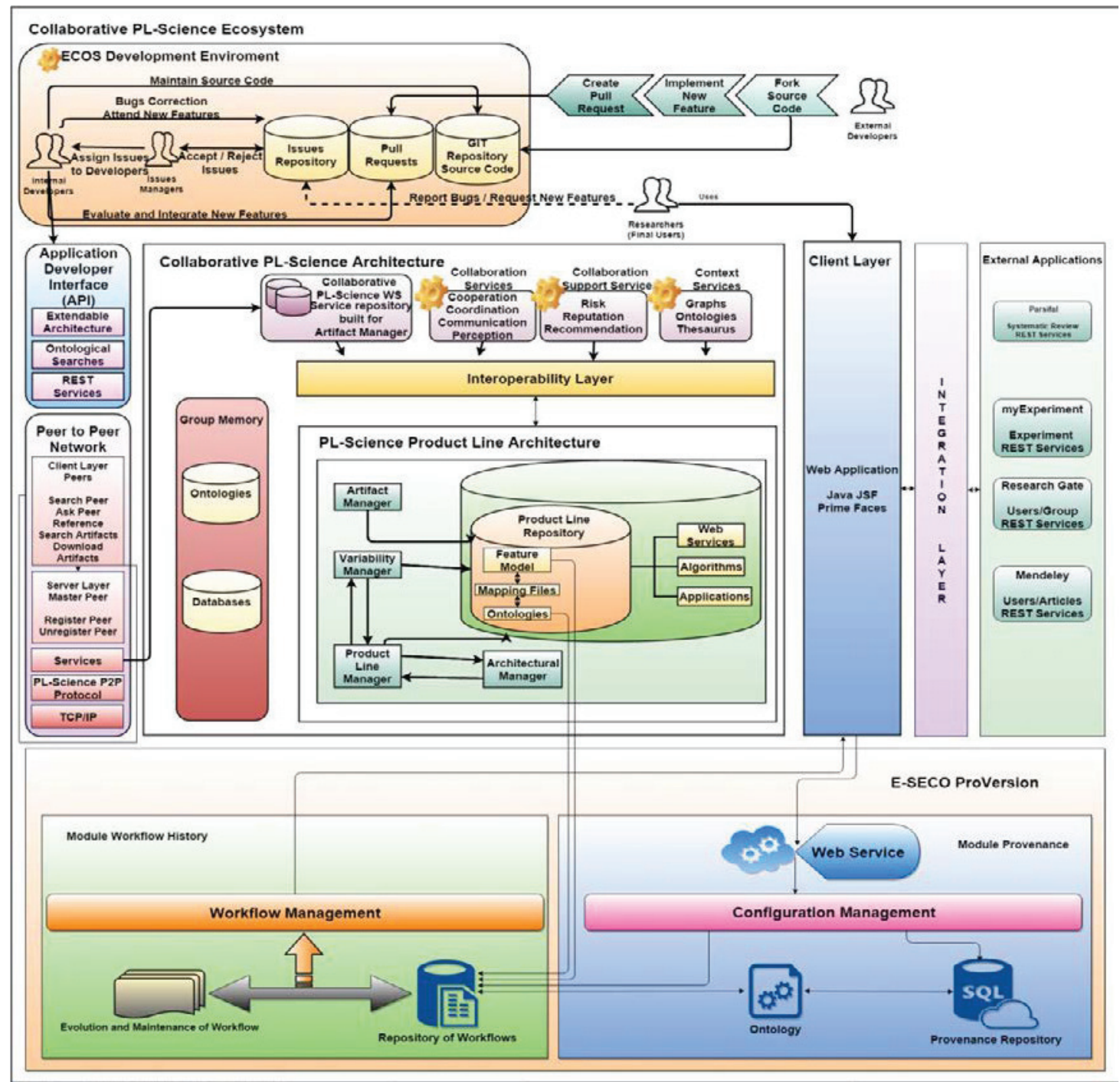

Figura 2. Arquitetura do E-SECO ProVersion. 


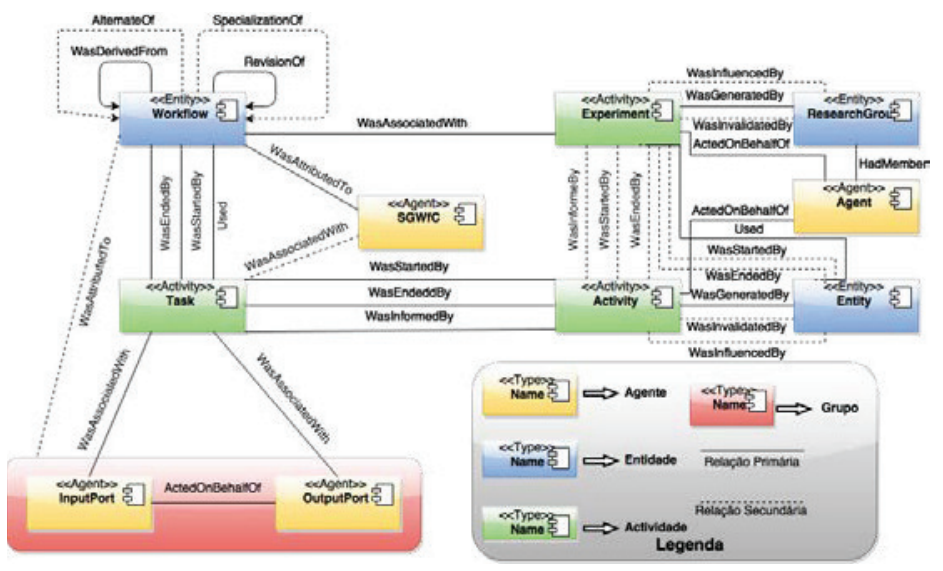

Figura 3. Relações causais da ontologia PROV-OEXT.

Além disso, no contexto de manutenção e evolução de software, uma fonte importante de informação são os dados históricos. A falta deste tipo de informação dificulta a análise dos resultados do experimento, o que pode impedir ou atrapalhar a reutilização dos workflows e dos experimentos, visto que não se conhece claramente a origem dos mesmos. Com o objetivo de mapear dados históricos, extraídos de repositórios existentes, foi realizado um estudo nos repositórios de workflows disponíveis, a fim de analisar à manutenção e evolução dos workflows. O referido estudo foi realizado junto aos repositórios myExperiment e CrowDLabs, ao longo do mês de outubro de 2015. No repositório CrowDLabs não foi possível extrair nenhum dado, devido a necessidade de permissão de acesso a base. No myExperiment constatouse que entre os 3692 workflows disponíveis na base, 1571 utilizam os SGWfCs Taverna, Kepler ou VisTrails, os quais são os principais SGWfC, representando mais de 40\% do total. Destes, 1520 são workflows desenvolvidos no SGWfC Taverna, 47 no SGWfC Kepler e 4 no VisTrails, destacando o Taverna como o principal em utilização entre os membros do repositório. Apesar de ser um número considerável, a quantidade é inferior a metade do total disponível no repositório, o que pode enviesar o estudo. Com relação ao histórico de versão dos workflows, observou-se que entre os 1571 workflows analisados, apenas $29 \%$ possuem dados de versionamento, o que reitera a falta de informações sobre o ciclo de vida do workflow, dificultando a sua reutilização.

Foi também realizada uma análise para a verificação das tarefas mais utilizadas entre os workflows estudados. Essa análise é útil por apresentar a quantidade de workflows que são afetados, caso uma tarefa seja modificada, o que irá impactar diretamente nos experimentos a que o workflow está vinculado. A lista das 10 tarefas mais utilizadas pode ser vista na Figura 4. Considerando a mineração de dados históricos, no repositório do myExperiment não existem informações sobre à proveniência dos workflows disponibilizados e os experimentos em que foram aplicados. Do ponto de vista da manutenção e evolução, tais informações se tornam essenciais para entender como os mesmos foram mantidos e evoluídos ao longo de um ciclo de experimentação.

A falta de dados históricos adequados dificulta a análise dos workflows e consequentemente dos experimentos pelo E-SECO ProVersion. Entretanto foi desenvolvido no contexto do E-SECO ProVersion um meta-repositório já preparado para extração e mineração destes dados de forma a contribuir com as pesquisas em e- 
Science. O objetivo é acessar repositórios de workflows existentes e complementar com informações necessárias para o controle de manutenção e evolução.

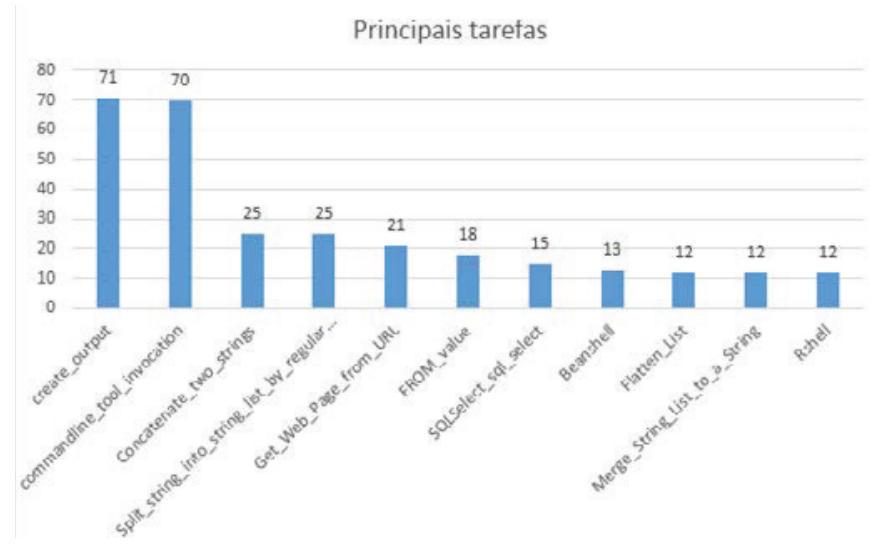

Figura 4. Lista das tarefas mais utilizadas nos workflows.

\section{Conclusão}

Atualmente, o E-SECO ProVersion permite ao pesquisador capturar os dados do workflow por meio de um serviço Web disponível na ferramenta. Os dados coletados junto aos workflows são referentes às atividades executadas, suas portas de comunicação, valores de entrada e saída, tempo de execução e informações sobre falhas na execução, além das relações causais entre as atividades. Estes dados alimentam a ontologia PROV-OEXT, que por meio de regras específicas do domínio detectam informações sobre a evolução e manutenção em workflows e experimentos, sendo essas informações disponibilizadas ao pesquisador por meio de uma interface Web.

A disposição destas informações ao pesquisador, de forma automatizada, facilita a identificação da origem das falhas e consequentemente a busca pela solução. Também contribuem para formação de uma base de conhecimento sobre o experimento, fortalecendo o uso de laboratórios colaborativos e trazendo ganhos ainda maiores no que tange a gerência de configuração dos experimentos.

\section{Agradecimentos}

Os autores agradecem o apoio da Fapemig, CAPES e CNPq.

\section{Referências}

Altintas, I., Berkley, C., Jaeger, E., Jones, M., Ludascher, B. and Mock, S. (2004). Kepler: an extensible system for design and execution of scientific workflows. In Scientific and Statistical Database Management. $16^{\text {th }}$ International Conference on (p. 423-424). IEEE.

Belloum, A., Inda, M. A., Vasunin, D., Korkhov, V., Zhao, Z., Rauwerda, H. and Hertzberger, L. O. (2011). Collaborative e-science experiments and scientific workflows. Internet Computing, IEEE, 15(4), p. 39-47.

Bosch, J. (2009, August). From software product lines to software ecosystems. In Proceedings of the 13th international software product line conference (pp. 111-119). Carnegie Mellon University.

Callahan, S. P., Freire, J., Santos, E., Scheidegger, C. E., Silva, C. T. and Vo, H. T. (2006). Managing the evolution of dataflows with VisTrails. In Data Engineering Workshops, 2006. Proceedings. $22^{\text {nd }}$ International Conference on (pp. 71-71). IEEE. 
Cuevas-Vicenttín, V., Kianmajd, P., Ludäscher, B., Missier, P., Chirigati, F., Wei, Y. and Dey, S. (2014). The PBase scientific workflow provenance repository. International Journal of Digital Curation, 9(2), 28-38.

Deelman, E., Gannon, D., Shields, M. and Taylor, I. (2009). Workflows and e-Science: An overview of workflow system features and capabilities. Future Generation Computer Systems, 25(5), p. 528-540.

Freitas, V.; David, J. M. N.; Braga, R. M. and Campos, Fernanda. (2015). An architecture for a Scientific Ecossystem. In: ${ }^{\text {th }}$ Workshop on Distributed Software Development, Software Ecosystems and Systems-of-Systems (WDES 2015), Belo Horizonte, Brazil, SBC, p. 41-48.

Gil, Y., Ratnakar, V., Deelman, E., Mehta, G.and Kim, J. (2007, July). Wings for pegasus: Creating large-scale scientific applications using semantic representations of computational workflows. In Proceedings of the National Conference on Artificial Intelligence (Vol. 22, No. 2, p. 1767).

Goble, C. A., Bhagat, J., Aleksejevs, S., Cruickshank, D., Michaelides, D., Newman, D. and De Roure, D. (2010). myExperiment: a repository and social network for the sharing of bioinformatics workflows. Nucleic acids research, 38(suppl 2), p. 677-682.

Hasan, R., Sion, R. and Winslett, M. (2007, October). Introducing secure provenance: problems and challenges. In Proceedings of the 2007 ACM workshop on Storage security and survivability (p. 13-18). ACM.

Lebo, T., Sahoo, S., McGuinness, D., Belhajjame, K., Cheney, J., Corsar, D. and Zhao, J. (2013). PROV-O: The PROV Ontology [www Document]. URL http://www.w3.org/TR/prov-o/

Lim, C., Lu, S., Chebotko, A., \& Fotouhi, F. (2010, July). Prospective and retrospective provenance collection in scientific workflow environments. InServices Computing (SCC), 2010 IEEE International Conference on (pp. 449-456). IEEE.

Mattoso, M., Werner, C., Travassos, G., Braganholo, V., Murta, L., Ogasawara, E. and Martinho, W. (2009). Desafios no apoio à composição de experimentos científicos em larga escala. Seminário Integrado de Software e Hardware, SEMISH, 9, 36.

Miranda, G; De Souza, J. A.; Braganholo, V.; Oliveira, D. de. (2014). CollabCumulus: Uma Ferramenta de Apoio à Análise Colaborativa de Proveniência em Workflows Científicos. SBSC.

Moreau, L. and Missier, P. (2013). PROV-DM: The PROV Data Model [www Document]. URL http://www.w3.org/TR/prov-dm/

Moreau, L., Clifford, B., Freire, J., Futrelle, J., Gil, Y., Groth, P. and Plale, B. (2011). The open provenance model core specification (v1.1). Future generation computer systems, 27(6), 743-756.

Oinn, T., Li, P., Kell, D. B., Goble, C., Goderis, A., Greenwood, M. and Zhao, J. (2007). Taverna/myGrid: aligning a workflow system with the life sciences community. In Workflows for e-Science (p. 300-319). Springer London.

Silva, V., Chirigati, F., Maia, K., Ogasawara, E., Oliveira, D., Braganholo, V. and Mattoso, M. (2010). SimiFlow: Uma Arquitetura para Agrupamento de Workflows por Similaridade. IV e-Science, 1-8.

Vaz, G. J., Giachetto, P. F., Torres, T. Z. and Massruhá, S. M. (2012). Um Modelo de Estrutura Organizacional em Plataformas de E-Science. In Anais do Congresso da Sociedade Brasileira de Computação (Vol. 32). 